\title{
5.
}

Ueber die Transcendenten, welche aus wiederholten Integrationen rationaler Formeln entstehen.

(Vom Herrn Prof. E. E. Kummer, Dr. phil. za Lieguitz.)

Die Reihen der reciproken Potenzzahlen von der Form $1+\frac{1}{2^{n}}+\frac{1}{3^{n}}+\ldots$ können, wie bekannt, wenn der Potenz-Exponent $n$ eine gerade Zahl ist, durch die Potenzen der Zahl $\pi$ summirt werden. Für ungerade PotenzExponenten aber hat man bis jetzt vergeblich versucht, dieselben durch bekannte Gröfsen auszudrücken. Es war mir nicht unwahrscheinlich, dafs sich auch diese Reihen durch die Zahl $\pi$ und durch Logarithmen würden summiren lassen. Ich unternahm deshalb, zunächst nur auf den angegebenen besonderen $Z_{w}$ eck ausgehend, eine Untersuchung der Reihe $\frac{x}{1^{2}}+\frac{x^{2}}{2^{3}}+\frac{x^{3}}{3^{3}}+\ldots$ Da diese Reihe durch das dreifache Integral $\int \frac{d x}{x} \int \frac{d x}{x} \int \frac{d x}{1-x}$ ausgedrückt wird, und da die Methoden, welche ich zur Untersuchung desselben anwendete, auch für bei weitem allgemeinere vielfache Integrale ausreichten, so stellte ich den hesonderen $\mathbf{Z}$ weck alsbald bei Seite, und ging an die allgemeine Untersuchung der Transcendenten, welche aus wiederholten Integrationen rationaler Formeln entstehen. Die erste Integration einer rationalen Formel $\int \boldsymbol{P} d x$ lälst sich bekanntlich immer vermittelst Logarithmen und Kreishogen ausführen. Multiplicirt man aber ein solches Integral wieder mit einer rationalen Function $Q$ und integrirt zum zweitenmale, so erhält man die Form $\int \boldsymbol{Q} \int \mathbf{P} d x . d x$. Die in dieser allgemeinen Form enthaltenen Transcendenten nenne ich logarithmische Integrale zweiter Ordnung. Wird die allgemeine Form dieser Integrale wieder mit einer rationalen Formel $\boldsymbol{R}$ multiplicirt und integrirt, so erhält man $\int \boldsymbol{R} \int \boldsymbol{Q} \int \boldsymbol{P} d x . d x . d x$, und ich nenne die in dieser allgemeinen Form enthaltenen Transcendenten logarithmische Integrale dritter Ordnung. Multiplicirt man immer wieder mit einer rationalen Formel und integrirt, so erbält man eben so die allgemeinen Formen der logarithmischen Integrale vierter, fünfter u. s. w. Ordnung. Die logarith- 
mischen Integrale erster Ordnung, welche nur Logarithmen und Kreisbogen sind, werden, als bekannt, übergangen. Ueber die logarithmischen Integrale zweiter Ordnung finden sich in Legendre Exercices de Calcul intégral nur einzelne Resultate, und erst in neuerer Zeit hat Hill diesélben zu einem besonderen Gegenstande von Untersuchungen gemacht. Dieser scharfsinnige Analytiker,", angereizt durch die glänzenden Erfolge, welche die Theorie der elliptischen Functionen, oder allgemeiner die Theorie der in der Form $\int \boldsymbol{P} \checkmark \boldsymbol{Q} d \boldsymbol{x}$ enthaltenen Transcendenten gehabt hat, hat eine ähnliehe Untersuchung der durch die allgemeinen Formen $\int \boldsymbol{P} \log \boldsymbol{Q} d \cdot \boldsymbol{x}$ und $\int \boldsymbol{P}$. Arc tang $\boldsymbol{Q} . d x$ ausgedrückten Transcendenten angestellt und hierüber zwei Abhandlungen herausgegeben. Die erste derselben ist in dem gegenw. Journale der Mathematik Bd.III. abgedruckt, die zweite aber, unter dem Titel Specimen exercitii analytici functionem integralem $\int \frac{d x}{x} \log \left(1+2 x \cos \alpha+x^{2}\right)$ tum quoad amplitudinem tum quoad modulum comparandi modum exhibentis, Londini Gothorum 1830, scheint, als akademische Gelegenheitsschrift, nur wenig bekannt zu sein. Eine neue Behandlung dieser logarithmischen Integrale zweiter Ordnung wird den ersten Theil der gegenwärtigen Abhandlung ausmachen. Obgleich nämlich Hill in seiner zweiten Abhandlung die eine dieser Transcendenten so vollständig behandelt hat, dafs wir, auf die wesentlichsten Eigenschaften derselben uns beschränkend, für dieselbè nur wenig neues hinzufügen können, so erschien uns dennoch auch diese einer neuen Behandlung werth, weil die von Hill gefundenen Resultate sich alle noch aufserordentlich vereinfachen lassen, und weil nach der Methode, welche wir zum Grunde legen, die Resultate, die bei Hill vereinzelt dastehen und fast alle nicht sowohl entwickelt, als vielmehr nur aufgestellt und mit besonderen Beweisen versehen werden, aus einer gemeinschaftlichen Quelle abgeleitet werden können. Aufserdem wird eiue neue Behandlung diẹser Transcendenten durch die Wichtigkeit des Gegenstandes gerechtfertigt. Denn wenn wir gleich nicht so weit gehen wie Hill, welcher diese Theorie für wichtiger und nützlicher hält als die Theorie der elliptischen Functionen, so glauben wir doch, dafs aus derselben der Analysis eine schöne Bereicherung erwachse. Der zweite Theil der gegenwärtigen Abhandlung wird die Theorie der logarithmischen Integrale dritter Ordnung enthalten. Für diese ist bisher noch fast gar nichts gethan worden, so dafs, mit Ausnahme geriuger Einzelheiten, alles was wir 
über dieselben sagen werden, für neu zu erachten ist. Für die logarith mischen Integrale höherer Ordnungen lassen wir die Allgemeinheit der Untersuchung fallen, da eine nur einigermafsen vollständige Theorie derselben uns zu weit führen würde, und da auch die Formeln; welche die Eigenschaflen dieser logarithmischen Integrale ausdrücken, für jede höhere Ordnung bedeutend weitläuftiger werden. Deshalb beschränken wir uns hier darauf, für die logarithmischen' Integrale vierter und fünfter Ordnung nur die Grundeigenschaften der einfachsten in ihnen enthaltonen Transcendenten zu entwickeln, dre mit der Reihe $\frac{x}{1^{n}}+\frac{x^{2}}{2^{n}}+\frac{x^{3}}{3^{n}}+\ldots$. in einem solchen Znsammenhange stehen, dats die gefundenen Eigenschaften derselben sich auch als Eigenschaften dieser merkwürdigen Reike darstellen lassen.

\section{Erster Theil.}

\section{Ueber die logarithmischen Integrale zweiter Ordnung.}

\section{1.}

Logarithmische Integrale zweiter Ordnung sind nach unserer Erklärung die in der allgemeinen Form $\int Q \int P . d x \cdot d x$, wo $P$ und $Q$ rationale Functionen von $x$ sind, enthaltenen Transcendenten; mit Ausschlufs der Logarithmen und Kreisbogen. Darum haben wir zunächst diese allgemeine Form in ihre einfachsten Bestandtheile zu zerlegen und so die einfachsten Formen der logarithmischen Integiale zweiter Ordinung zu suchen. Es kann zunächst angenommen werden, dafs $\boldsymbol{P}$ und $\boldsymbol{Q}$ rationale gebrochene Functionen von $x$ sind, von der Art, dafs sie keine ganzen Theile enthalten, oder, was dasselbe ist, dafs in den Nennern derselben höhere Potenzen von $x$ vorkommen als in den Zählern; denn ist dies nicht der Fall, so kann man die ganzen Theile davon absondern und $q+\boldsymbol{Q}$ stalt $\boldsymbol{Q}$ und

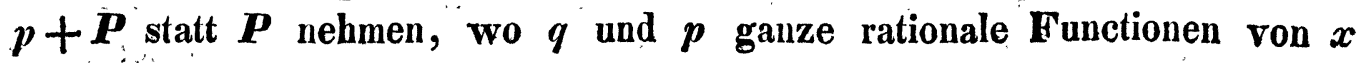
sind; dadurch zerfällt dann das obige Integral in folgende vier:

$$
\int q \int p d x . d x, \int q \int P d x . d x, \int Q \int p d x . d x, \int Q \int P d x . d x
$$

Da nun das Integral emer ganzen rationalen Function von $x$ wieder eine solche Function ist, so "ist klar, dafs das erste Integral nur eine ganze 
Function giebt, und dafs das zweite und dritte nur Logarithmen und Kreisbogen enthalten: Es bleibt daher nur das vierte Integral übrig, in welchem $\boldsymbol{P}$ und $\boldsymbol{Q}$ keine ganzen Theile mehr enthalten. Werden nun $\boldsymbol{P}$ und $\boldsymbol{Q}$ in Partialbrüche von der Form $\frac{a}{(b+c x)^{m}}$ zerlegt, wo $a, b, c$ auch imaginär sein kŏnnen und $m$ eine ganze positive $Z$ ahl ist, so zerfältt das allgemeine Integral von selbst in eine Summe mehrerer einzelner Integrale von der Form

$$
\int \frac{a \cdot d x}{(b+c x)^{m}} \int \frac{e d x}{(f+g x)^{n}}
$$

Dieses Integral kann aber, wenn $m$ und $n$ nicht beide zugleich der Einheit gleich sind, immer rational oder durch Logarithmen und Kreisbogen integrirt werden, so dafs wieder nur der Fall $m=1$ und $n=1$ zu betrachten übrig bleibt. Für diesen Fall aber erhält man durch Ausführung der ersten Integration folgende Form:

$$
\int \frac{l(f+g x) d x}{b+c x}
$$

Setzt man jetzt, um zu vereinfachen,

$$
f+g x=k z, \quad k=\frac{b g-f c}{c},
$$

so wird $b+c \cdot x=\frac{c k(1+z)}{g}$, und das Integral geht in folgende zwei über:

$$
\frac{a}{c} \int \frac{l z d z}{1+z}+\frac{a l(k)}{c} \int \frac{d z}{1+z} .
$$

Da das zweite dieser Integrale nur einen Logarithmus giebt, so ist das Integral $\int \frac{l z \cdot d z}{1+z}$ im Grunde das einzige in der obigen allgemeinen Form enthaltene. Da aber $z$ auch imaginär sein kann, so zerfällt dasselbe, wie wir alsbald zeigen werden, durch die Sonderung des realen und imaginären Theiles, in zwei verschiedene. Es ist zweckmäfsig, für dieses Integral ein besonderes Functionszeichen einzuführen, welches demselben namentlich dann zukommt, wenn $z$ real ist; wo dann der Buchstabe $x$ dafür gesetzt werden soll. Da ferner $l x$ nur für positive Werthe des $x$ real sein würde, so wollen wir dafür $l( \pm x)$ setzen, unter der Bedingung, dals $\pm x$ immer positiv zu nehmen sei; denn die Bedingung, dafs $x$ nur positiv sein darf, würde den bald zu entwickelnden Formeln eine störende Beschränkung 
auferlegén *). Als Fưnctionszeichen fár dieses Integral wähle ich den Buclistaben $\mathcal{A}$, und nohme das Integral so, dars es zugleich mit $x$ verschwindet, also so, dafs.

$$
A(x)=\int_{\sigma}^{x} \frac{l( \pm x) d x}{1+x}
$$

Wenn aber $z$ imaginär ist; so nehme ich $z=x \xi^{\alpha i}$, wo $x$ und a real sind und $i=\sqrt{-1}$ ist. Hierdurch wird dieses Integral

$$
\int \frac{(l( \pm x)+\alpha i) e^{\alpha i} d x}{1+x e^{\alpha i}}
$$

oder, durch Trennung der realen und imaginären Theile,

$$
\int \frac{(x+\cos \alpha) l( \pm a)-\alpha \sin \alpha}{1+2 x \cos \alpha+x^{2}}+i \int \frac{\sin \alpha l( \pm x)+\alpha(x+\cos \alpha)}{1+2 x \cos \alpha+x^{2}} d x .
$$

Sondert man hiervon wieder diejenigen Theile $a b$, welche durch Logarithmen und Kreisbogen sich integriren lassen, so bleiben nur die beiden Integrale

$$
\int \frac{l( \pm x)(x+\cos \alpha) d x}{1+2 x \cos \alpha+x^{2}} \text { und } \int \frac{l( \pm x) \cdot \sin \alpha \cdot d \alpha}{1+2 x \cos \alpha+x^{2}}
$$

als die einzigen realen Integrale übrig, welche in der oben aufgestellten allgemeinen Form enthalten sind; denn das Integral $A(x)$ ist nur ein specieller Fall des ersteren von diesen, welchen man erhält, wenn man $\alpha=0$ nimmt. Wir nehmen auch diese Integrale so, dafs sie zugleich mit $x$ verschwinden und bezeichnen dieselben, als Functionen zweier veränderlichen Grölsen, durch $\boldsymbol{D}(x, \alpha)$ und $\boldsymbol{E}(x, \alpha)$, so dafs

$$
\begin{aligned}
& \boldsymbol{D}(x, \alpha)=\int_{0}^{x} \frac{l( \pm x)(x+\cos \alpha) d x}{1+2 x \cos \alpha+x^{2}} \\
& \boldsymbol{E}(x, \alpha)=\int_{0}^{x} \frac{l( \pm x) \cdot \sin \alpha \cdot d x}{1+2 x \cos \alpha+x^{2}} .
\end{aligned}
$$

Die imaginäre Function $A\left(x e^{\alpha i}\right)$ wird nun durch die Functionen $\boldsymbol{D}(x, \alpha)$ und $\boldsymbol{E}(\boldsymbol{x}, \alpha)$ auf folgende Art ausgedrückt:

$A\left(x e^{\alpha i}\right)=D(x, \alpha)-\alpha \operatorname{Arctang} \frac{x \sin \alpha}{1+x \cos \alpha}+i E(x, \alpha)+\frac{i \alpha}{2} l\left(1+2 x \cos \alpha+x^{2}\right)$.

Es hat jetzt durchaus keine Schwierigkeiten, irgend ein gegebenes Integral von der Form $\int \boldsymbol{P} \int \boldsymbol{Q} d x d x$, unter welche Form auch die von Hill ge-

*) Ueberall wo Logarithmen aus der Integration rationaler Formeln entstehen, wie es hier durchgehends der Fall ist, kann man unter dem Logarithmenzeichen mit gleichem Rechte beide Vorzeichen + und - gelten lassen, und diese sind dann, wo es sich um reale Gröfsen handelt, so zu bestimmen, dafs die Quantität, deren Logarithmus zu nehmen ist, immer positiv sei. Deshalb ersuchen wir den Leser, zu den sämmtlichen Logarithmenzeichen, welche in dieser Abhandlung vorkommen werden, sich immer das Zeichen \pm hinzuzudenken und es so zu bestimmen, dafs dadurch die Grölse, vor welcher es steht, positiv werde. 
wählten Formen $\int P \log Q d x$ und $\int P$ Arctang $Q d x$ gehören, durch rationale Functionen, durch Logarithmen und durch die beiden Functionen $\boldsymbol{D}(\boldsymbol{x}, \alpha)$ und $\boldsymbol{E}(x, \alpha)$ zu integriren. Zu diesem $\mathbf{Z}$ wecke darf man nur, wie wir es gezeigt haben, durch Zerlegung in Partialbrüche das Integral in Theile zerlegen, welche sich rational oder nur durch Logarithmen und durch die Function $A$ integriren lassen. Die Functionen $A$, welche unmögliche Grörsen enthalten, zerlegt man alsdann nach der hier gegebenen Formel in Functionen $D$ und $\boldsymbol{E}$ und Logarithmen und Kreisbogen; und eben so zerlegt man, nach bekannten Formeln, die unmöglichen Logarithmen in Logarithmen und Kreisbogen: alsdann verschwinden die unmöglichen GröIsen von selbst und man hat die verlangte Integration ausgeführt.

Die beiden Integrale $\boldsymbol{D}(x, \alpha)$ und $\boldsymbol{E}(x, \alpha)$ nehmen noch eine einfachere Form an, wenn statt $x$ eine andere veränderliche Grölse $u$ eingeführt wird, welche durch die Gleichung

bestimmt ist. Hierdurch wird nämlich

$$
\operatorname{tang} u=\frac{-x \sin \alpha}{1+x \cos \alpha} \quad \text { oder } \quad x=\frac{-\sin u}{\sin (u+\alpha)}
$$

$$
\begin{aligned}
& \boldsymbol{D}\left(\frac{-\sin u}{\sin (u+\alpha)}, \alpha\right)=-\int_{0} l\left(\frac{ \pm \sin u}{\sin (u+\alpha)}\right) \operatorname{cotang} u \cdot d u, \\
& \boldsymbol{E}\left(\frac{-\sin u}{\sin (u+\alpha)}, \alpha\right)=-\int_{0} l\left(\frac{ \pm \sin u}{\sin (u+\alpha)}\right) d u .
\end{aligned}
$$

Diese Substitution zeigt auch, dafs das Integral $\boldsymbol{E}(x, a)$ sich in einfachere, nur von einem Elemente abhängige Integrale von der Form $\int l( \pm \sin u) d u$ zerlegen läfst. Diese Integrale aber lassen sich wieder durch die specielle Function $\boldsymbol{E}(-1, \alpha)$ ausdrücken, so dafs die allgemoine Function $\boldsymbol{E}(\boldsymbol{x}, \alpha)$ sich immer durch Functionen derselben Art ausdrücken lälst, in welchen das erste Element den bestimmten Werth -1 hat. Um dies zu zeigen differenziire man $\boldsymbol{E}(x, \alpha)$ in Beziehung auf $\alpha$, welches

$$
\frac{d E(x, \alpha)}{d \alpha}=\frac{x(x+\cos \alpha) l( \pm x)}{1+2 x \cos \alpha+x^{2}}-\frac{1}{2} l\left(1+2 x \cos \alpha+x^{2}\right)
$$

giebt. Hieraus folgt für $x=-1$

$$
\frac{d E(-1, \alpha)}{d \alpha}=-\frac{1}{2} l(2-2 \cos \alpha)=-l\left(2 \sin \frac{1}{2} \alpha\right),
$$

also, durch Integration,

$$
E(-1, \alpha)=-\int l\left(\sin \frac{1}{2} \alpha\right) \cdot d \alpha-\alpha l 2+\text { const. }
$$

und, wenn $\alpha=2 u$ gesetzt wird,

$$
\int l(\sin u) d u=-\frac{1}{2} E(-1,2 u)-u l 2+\text { const. }
$$


I)a aber

so hat man

$$
E(x, \alpha)=-\int l(\sin u) \cdot d u+\int l \sin (u+\alpha) \cdot d u
$$

$$
\boldsymbol{E}(x, \alpha)=\frac{1}{2} \boldsymbol{E}(-1,2 u)-\frac{1}{2} \boldsymbol{E}(-1,2 u+2 \alpha)+\text { const. }
$$

und endlich, wenn die Constante durch $u=0$, und auch $x=0$, bestimmt wird, so hat man

$$
\begin{gathered}
E(x, a)=\frac{1}{2} \boldsymbol{E}(-1,2 u)+\frac{1}{2} \boldsymbol{E}(-1,2 \alpha)-\frac{1}{2} \boldsymbol{E}(-1,2 u+2 a) \\
\text { wenn } x=\frac{-\sin u}{\sin (u+\alpha)} .
\end{gathered}
$$

Da die Integrale $\boldsymbol{D}(x, \alpha)$ und $\boldsymbol{E}(x, \alpha)$ die einfachste Gestalt erhalten, wenn $x=\frac{-\sin u}{\sin (u+\alpha)}$ gesetzt wird, so werden wir in der Folge einfacher

$$
\begin{aligned}
& D\left(\frac{-\sin u}{\sin (u+\alpha)}, a\right) \text { durch } D(u, \alpha) \\
& E\left(\frac{-\sin u}{\sin (u+\alpha)}, \alpha\right) \text { durch } E(u, \alpha)
\end{aligned}
$$

bezeichnen. Da jedoch einige der zu entwickelnden Formeln sich leichter ausdrücken lassen, wenn dem $x$ sein ursprünglicher Werth gelassen wird, so werden wir auch ferner von den Functionen $\boldsymbol{D}(x, \alpha)$ und $\boldsymbol{E}(\boldsymbol{x}, \alpha)$ Gebrauch machen. Zu erinnern ist, dafs man nicht meinen müsse, $\boldsymbol{D}(x, \alpha)$ und $D(u, \alpha)$ seien einander gleich für $x=u$ : sie sind vielmehr einander gleich, wenn $x=\frac{-\sin u}{\sin (u+a)}$ genommen wird. Eben so ist es mit $\boldsymbol{E}(\boldsymbol{x}, \alpha)$ und $\boldsymbol{E}(\boldsymbol{u}, \alpha)$. Diese Function ist zwar durch die einfachere, nur von einem Elemente abhängige Function $\boldsymbol{E}(-1, a)$ überflüssig gemacht; da sie aber viele einfache Eigenschaften hat, welche denen der Function $\boldsymbol{D}(u, \alpha)$ analog sind, so werden wir auch sie beibehalten.

Die hier gegebene Zerlegung der allgemeinen Form des Integrales $\int \boldsymbol{P} \int \boldsymbol{Q} d x d \boldsymbol{x}$ in seine einfachsten Bestandtheile enthält ungefähr die Resultate der ersten Abhandlung von Hill; denn die daselbst untersuchten allgemeinen Formen $\int P l Q d x$ und $\int P$ Arctang $Q d x$ sind beide in der obigen Form enthalten. Den einfachsten Functionen aber, welche in dieser Form enthalten sind, haben wir etwas andere Gestalten geben müssen, nicht uur weil so die Eigenschaften derselben einfachere Ausurücke annehmen, sondern auch um die Analogie mit den später zu entwickelnden logarithmischen Integralen von höheren Ordnungen zu erhalten. 
5. 2.

Wir entwickeln nun zuerst die Grundformeln für die Function $\Lambda(x)$; deun wenn gleich dieselbe nur ein specieller. Fall von $D(x, \alpha)$ ist, so dient sie doch der ganzen Theorie der logarithmischen Integrale zweiter Ordnung zur Grundlage, da die Grunformeln der Functionen $\boldsymbol{D}(\boldsymbol{x}, \alpha)$ und $\boldsymbol{E}(x, a)$ sich leicht aus denen der Function $A(x)$ entwickeln lassen. Die im \$. 1. gezeigte Zerlegung des Integrals $\int P \int Q d x d x$ in seine einfachsten Bestandtheile giebt hier sogleich eine Methode, welche hinreicht, eine unendliche $Z$ ahl von Formeln für die Function $A(x)$ zu finden. Setzt man nämlich statt $x$ irgend eine rationale Function $\frac{p}{q}$ von der Art, dafs $p, q$ und $p+q$ nur reale Factoren ersten Grades enthalten, so hat man

$$
A\left(\frac{p}{q}\right)=\int \frac{l\left(\frac{p}{q}\right)(q \partial p-p \partial q)}{q(q+p)} .
$$

Zerlegt man nun dieses Integral nach der oben gezeigten Methode in seine einfachen Bestandtheile, so erhält man $\Lambda\left(\frac{p}{q}\right)$ ausgedrückt durch ein Aggregat derselben Functionen $A$ und durch Logarithmen, welche alle real sind, indem wir vorausgesetzt haben, dafs $p, q$ und $p+q$ nur reale Factoren ersten Grades enthalten sollen. Die einfachsten Formeln dieser Art wird man unstreitig erhalten, wenn man für $p$ und $q$ ganze rationale Functionen ersten Grades nimmt. Es sei deshalb $p=a+b x, q=c+d x$, so wird

und da

$$
A\left(\frac{a+b x}{c+d x}\right)=\int l\left(\frac{a+b x}{c+d x}\right) \frac{(b c-a d) \partial x}{(c+d x)(a+c+(b+d) x)},
$$

$$
\frac{b c-a d}{(c+d x)(a+c+(b+d) x)}=\frac{b+d}{a+c+(b+\bar{d}) x}-\frac{d}{c+d x}
$$

so wird dieses Integral in folgende vier zerlegt:

$$
\begin{aligned}
A\left(\frac{a+b x}{c+d x}\right) & =\int \frac{l(a+b x) \cdot(b+d) \partial x}{a+c+(b+d) x}-\int \frac{l(c+d x)(b+d) \partial x}{a+c+(b+d) x} \\
& -\int \frac{l(a+b x) \cdot d \cdot \partial x}{c+d x}+\int \frac{l(c+d x) \cdot d \cdot \partial x}{c+d x} .
\end{aligned}
$$

Drückt man diese vier Integrale einzeln durch die Function $A$ und durch Logarithmen aus, so erhält man, nach einigen leichten Reductionen des logarithmischen Theiles,

$$
\begin{gathered}
A\left(\frac{a+b x}{c+d x}\right)=A\left(\frac{(b+d)(a+b x)}{b c-a d}\right)-A\left(\frac{-(b+d)(c+d x)}{b c-a d}\right) \\
-\Lambda\left(\frac{d(a+b x)}{b c-a d}\right)+\frac{1}{2}\left(l\left(\frac{d(c+d x)}{b c-a d}\right)\right)^{2}+\text { Const. }
\end{gathered}
$$

Crelle's Joumal d. M. Bd. XXI. Hft. 1. 
Die grofse Allgemeinheit dieser Formel, welche fünf von einander unabhängige, nach Belieben zu bestimmende Grölsen enthält, ist nur scheinbar, da die Anzahl dieser Gröfsen durch passende Substitutionen sich auf zwei einschränken läfst. Setzt man nämlich $\frac{d(a+b x)}{b c-a d}=-z, \frac{b+d}{d}=y$, so erhält die Formel die einfachere Gestalt:

$$
A\left(\frac{z(1-y)}{1-z}\right)=A(-y z)-A\left(\frac{y(1-z)}{1-y}\right)-A(-z)+\frac{1}{2}\left(l \frac{1-z}{1-y}\right)^{2}+\text { Const. }
$$

Nimmt man Const. $=-A(-y)-C$ und verwandelt wieder $z$ in $x$, so kann man die Formel auch so darstellen:

$$
A(-x y)=A(-x)+A(-y)+A\left(\frac{x(1-y)}{1-x}\right)+A\left(\frac{y(1-x)}{1-y}\right)-\frac{1}{2}\left(l \frac{1-x}{1-y}\right)^{2}+C .
$$

Die Constante $\boldsymbol{C}$ in dieser Formel ist von $\boldsymbol{x}$ unabhängig. Da man aber $x$ und $y$ mit einander vertauschen kann, ohne dafs die Formel sich änderte, so mufs diese Constante auch von $y$ unabhängig sein, und deshalb ist sie rein numerisch. Ehe wir diese Constante allgemein bestimmen, wollen wir den speciellen Fall betrachten, wo $y=x$ ist. Für diesen geht die Gleichung über in

$$
\Lambda\left(-x^{2}\right)=2 \Lambda(-x)+2 \Lambda(x)+C .
$$

Setzt man zur Bestimmung der Constante $x=0$, so erhält man $C=0$; und dieser Werth mufs in dem ganzen Intervalle von $x=-\infty$ bis $x=+\infty$ gültig sein, weil in demselben keine Discontinuität eintritt. Man hat daher

$$
\text { 1. } . \Lambda\left(-x^{2}\right)=2 \Lambda(-x)+2 \Lambda(x) \text {. }
$$

Um nun die Constante der allgemeineren Formel zu bestimmen, mufs man zuuächst bemerken, dafs die Continuität der darin vorkommenden Functionen unterbrochen wird, sobald 1-x oder 1-y aus dem Positiven in's Negative übergeht, und umgekehrt; sobald aber die Continuität unterbrochen wird, kann auch die Constante der Integration plötzlich ihren Werth ändern. Deshalb sind hier vier Fälle zu unterscheiden, für welche die Constante besonders zu bestimmen ist; 1) wenn $1-x$ positiv und $1-y$ positiv, 2) wenn $1-x$ positiv und $1-y$ negativ, 3) weun $1-x$ negativ und $1-y$ positiv, 4) wenn $1-x$ negativ und $1-y$ negativ ist. In dem ersten Falle findet man, indem man $x=0$ and $y=0$ setzt, auch $C=0$. In dem zweiten Falle setze man $x=0$ und $y=2$, so wird $C=-2 A(-2)$, und man findet denselben Werth der Constante für den dritten Fall, wenn man $x=2$ und $y=0$ setzt. Um endlich die Constante für den vierten Fall zu bestimmen setze man $x=2$ und $y=2$, wodurch man erhält 
$A(-4)=2 A(-2)+2 \Lambda(2)+C$. Dieser Werth der Constante wird vermöge Formel (1.) zu $\boldsymbol{C}=0$. Die Constante der allgemeinen Formel hat also nur die beiden verschiedenen Werthe $C=0$ und $C=-2 A(-2)$, und zwar den ersten, wenn $1-x$ und $1-y$ gleiche Vorzeichen haben, den zweiten, wenn die Vorzeichen dieser Grörsen verschieden sind, oder, was dasselbe ist: er ist $C=0$ wenn $\frac{1-x}{1-y}$ positiv und $C=-2 A(-2)$ wenn $\frac{1-x}{1-y}$ negativ ist. Setzt man in der allgemeinen Formel für den zweiten dieser Fälle $1-x=+w$ und $1-y=-w$ und nimmt $w$ unendlich klein, so erbält man $C=-3 \Lambda(-1)$. Es ist daher $2 \Lambda(-2)=3 \Lambda(-1)$, und da $\Lambda(-1)=1+\frac{1}{2^{2}}+\frac{1}{3^{2}}+\ldots=\frac{\pi^{2}}{6}$, so ist $C=-\frac{\pi^{2}}{2}$. Wir haben daher folgende Grundformel für die Function $A$ :

\section{2. $A(-x y)$}

$$
=A(-x)+A(-y)+A\left(\frac{x(1-y)}{1-x}\right)+A\left(\frac{y(1-x)}{1-y}\right)-\frac{1}{2}\left(l \frac{1-x}{1-y}\right)^{2}+C ;
$$

wo $C=0$ wenn $\frac{1-x}{1-y}$ positiv und $C=-\frac{\pi^{2}}{2}$ wenn $\frac{1-x}{1-y}$ negativ ist.

Diese Formel stimmt mit derjenigen überein, welche Hill in seiner zweiten Abhandlung aufgestellt und durch Differenziiren bewiesen hat. Da aber nach der von Hill gewählten Definition der Function $\Lambda(x)$ dieselbe imaginär wird, sobald $1-x$ negativ ist, so konnte er nur den einen Fall betrachten, wo $1-x$ und $1-y$ beide positiv sind. In dieser Formel sind alle bisher bekannten Eigenschaften der Function $A(x)$ als specielle Fälle enthalten; welche wir jetzt aus derselben ableiten wollen.

Setzt man $y=\frac{1}{x}$ und verwandelt sodann $x$ in $-x$, so erhält man

$$
\text { 3. } \quad A(x)+\Lambda\left(\frac{1}{x}\right)=\frac{1}{2}(l \pm x)^{2}+K \text {; }
$$

wo $K=-\frac{\pi^{2}}{8}$ wenn $x$ positiv und $K=\frac{\pi^{2}}{3}$ wenn $x$ negativ ist. Setzt man $y=0$, so erhält man

$$
\text { 4. } A(-x)+A\left(\frac{x}{1-x}\right)=\frac{1}{2}(l(1-x))^{2}+K \text {; }
$$

wo $K=0$ wenn $1-x$ positiv und $K=\frac{\pi^{2}}{2}$ wenn $1-x$ negativ ist. Verwandelt man hierin $x$ in $1-x$, addirt die so erhaltene Formel zu jener und verwandelt die Summe der beiden Functionen $A\left(\frac{x}{1-x}\right)+A\left(\frac{1-x}{x}\right)$ nach Formel (3), so erhält man

5. $A(-x)+A(x-1)=l x l(1-x)+\frac{\pi^{2}}{6}$. 
Subtrahirt man ferner (4) und (5) von einander und verwandelt $x$ in $1-x$, so hat man

$$
\text { 6. } \quad \Lambda(-x)-\Lambda\left(\frac{1-x}{x}\right)=l x l(1-x)-\frac{1}{2}(l x)^{2}+K
$$

wo $K=\frac{\pi^{2}}{6}$ wenn $x$ positiv und $K=-\frac{\pi^{2}}{3}$ wenn $x$ negativ ist. Verwandelt man endlich hierin wieder $x$ in $\frac{1}{1-x}$, so hat man

$$
\text { 7. } A(-x)-A\left(\frac{-1}{1-x}\right)=l x l(1-x)-\frac{1}{2}(l(1-x))^{2}+K
$$

wo $K=-\frac{\pi^{2}}{6}$ wenn $1-x$ positiv and $K=+\frac{\pi^{2}}{3}$ wenn $1-x$ negativ ist.

Diese Gleichungen (3 bis 7), welche wir aus der allgemeinen Formel (2) abgeleitet haben, enthalten die einfachsten Eigenschaften der Function $\Lambda(x)$, indem sie nur zwei solche Functionen mit einander verbinden, deren Summe oder Differenz sich durch Logarithmen ausdrücken läfst. Durch dieselben kann man aus dem einen bekannten Werthe der Function $\Lambda(-1)=\frac{\pi^{2}}{6}$ noch mehrere andere Werthe dieser Function ableiten. Setzt man in Formel (1) $x=1$, so hat $\operatorname{man} 2 A(+1)+\Lambda(-1)=0$, also $A(+1)=-\frac{\pi^{2}}{12}$. Setzt man weiter in der Formel (5) $x=2$, so hat $\operatorname{man} A(-2)=$ $\frac{\pi^{2}}{6}-\Lambda(+1)$, also $\Lambda(-2)=\frac{\pi^{2}}{4}$. Setzt man endlich $x=\frac{1}{2}$ in Formel (5), so ist $A\left(-\frac{1}{2}\right)=\frac{1}{2}(\Lambda 2)^{2}+\frac{\pi^{2}}{12}$. Aufser diesen lassen sich noch einige andere besondere Werthe der Function $A(x)$ durch Logarithmen und durch die Zahl $\pi$ ausdrücken. Nimmt man nämlich $x=\frac{\sqrt{5}-1}{2}$, welchen Werth ich kurz durch $r$ bezeichne, so ist $r^{2}=1-x, \frac{1-r}{r}=r$ : also erhäl man: durch Formel (1) und (5)

$$
\begin{gathered}
A\left(-r^{2}\right)=2 A(-r)+2 A(r) \\
A(-r)+A\left(-r^{2}\right)=2(l r)^{2}+\frac{\pi^{2}}{6}
\end{gathered}
$$

woraus durch Elimination des $\Lambda^{\prime}\left(-r^{2}\right)$ folgt:

$$
3 A(-r)+2 A(r)=2(l r)^{2}+\frac{\pi^{2}}{6}
$$

Ferner giebt Formel (6), wenn $x=r$ gesetzt wird,

$$
A(-r)-A(r)=\frac{3}{2}(l r)^{2}+\frac{\pi^{2}}{6}
$$


und aus diesen beiden Gleichungen erhält man sogleich die Werthe von $\Lambda(-\boldsymbol{r})$ und $A(+\boldsymbol{r})$, nämlich:

$$
A\left(\frac{V 5-1}{2}\right)=-\frac{1}{2}\left(l \frac{V 5-1}{2}\right)^{2}-\frac{\pi^{2}}{15}, A\left(\frac{1-V 5}{2}\right)=\left(l \frac{V 5-1}{2}\right)^{2}+\frac{\pi^{2}}{10} .
$$

Hieraus aber kann man durch Anwendung der Formeln (3 bis 7) leicht folgende ableiten :

$$
\begin{aligned}
& A\left(\frac{\sqrt{5}-3}{2}\right)=\left(l \frac{\sqrt{5}-1}{2}\right)^{2}+\frac{\pi^{2}}{15}, A\left(\frac{-\sqrt{5}-3}{2}\right)=\left(l \frac{\sqrt{5}-1}{2}\right)^{2}+\frac{4 \pi^{2}}{15}, \\
& A\left(\frac{\sqrt{5}+1}{2}\right)=\left(l \frac{\sqrt{5}-1}{2}\right)^{2}-\frac{\pi^{2}}{10}, A\left(\frac{-\sqrt{5}-1}{2}\right)=-\frac{1}{2}\left(l \frac{\sqrt{5}-1}{2}\right)^{2}+\frac{7_{\pi}}{30} .
\end{aligned}
$$

Einige andere Formeln, welche als specielle Fälle in der Formel (2) enthalten sind, in denen aber mehr als zwei Functionen $A$ vorkommen, sind folgende:

$$
\text { 8. } \quad \Lambda(-4 x(1-x))=2 \Lambda(-2 x)+2 \Lambda(2 x-2)-\frac{\pi^{2}}{2} \text {. }
$$

9. $A(-x(1-x))=A\left(\frac{x^{2}}{1-x}\right)-A\left(\frac{x}{(1-x)^{2}}\right)-2 l x l(1-x)+\frac{3}{2}(l(1-x))^{2}+K$; wo $K=0$ wenn $1-x$ positiv und $K=-\frac{\pi^{2}}{2}$ wenn $1-x$ negativ ist. 10. $A\left(\frac{x^{2}}{4-4 x}\right)=2 A\left(\frac{-x}{2}\right)+2 A\left(\frac{x}{2-2 x}\right)-\frac{1}{2}(l(1-x))^{2}+K$; wo $K=0$ wenn $1-x$ positiv und $K=-\frac{\pi^{2}}{2}$ wenn $1-x$ negativ ist. 11. $A\left(x^{2}\right)-\frac{1}{2} A\left(-x^{2}\right)=A\left(\frac{x(1+x)}{1-x}\right)+A\left(\frac{-x(1-x)}{1+x}\right)-\frac{1}{2}\left(l \frac{1-x}{1+x}\right)^{2}+K$; wo $K=0$ wenn $\frac{1-x}{1+x}$ positiv und $K=-\frac{\pi^{2}}{2}$ wenn $\frac{1-x}{1+x}$ negativ ist.

Die Formel (8) ist aus (2) entstanden, indem $x$ in $2 x$ verwandelt und $y=2-2 x$ gesetzt worden ist, und die Formel (9) durch den besonderen Werth $y=1-x$, mit Zuziehung der Formeln (3) und (5). Ferner ist (10) aus (2) entstander, indem $x$ in $\frac{x}{2}$ verwandelt und $y=\frac{-x}{2-2 x}$ gesetzt worden ist, und Formel (11) durch den besonderen Werth $y=-x$, mit Anwendung von Formel (1). Uebrigens ist klar, dafs sich diese Formeln aufserordentlich vervielfältigen lassen, da dem $y$ in der allgemeinen Formel alle beliebigen Werthe gegeben werden können. Man kann aber auch die in diesen Formeln vorkommenden Functionen $A$ mit Hülfe der obigen füıf einfachen Gleichungen umformen, und diese Verwandlungen lassen sich auch mit der allgemeinen Formel (2) selbst vornehmen, so dals sie 
viele verschiedene Gestalten annimmt. Verwandelt man z. B. die vierte und fünfte Function $A$ dieser Formel nach Formel (4), so erhält man

12. $\quad A(-x y)=$

$A(-x)+A(-y)-A\left(\frac{-x(1-y)}{1-x y}\right)-A\left(\frac{-y(1-x)}{1-x y}\right)+l\left(\frac{1-x}{1-x y}\right) \cdot l\left(\frac{1-y}{1-x y}\right)+K$, wo immer $K=0$, mit Ausnahme des Falles, dafs 1-x und 1-y gleiche Vorzeichen haben und 1- $x y$ das entgegengesetzte von dem weun $K=\pi^{2}$ ist.

Besonders merkwürdig ist folgende Umgestaltung der Formel (2), bei welcher der logarithmische Theil ganz verschwindet. Um dieselbe zu erhalten verwandle man in der Formel (2) zunächst $y$ in $\frac{1}{y}$. Dieses giebt

$$
\begin{gathered}
A\left(-\frac{x}{y}\right)= \\
\Lambda(-x)+\Lambda\left(-\frac{1}{y}\right)+A\left(-\frac{x(1-y)}{y(1-x)}\right)+\Lambda\left(-\frac{1-x}{1-y}\right)-\frac{1}{2}\left(l \frac{y(1-x)}{1-y}\right)^{2}+C .
\end{gathered}
$$

Ferner verwandle man in dieser Formel wieder $x$ in $\frac{-x(1-y)}{1-x}$ und $y$ in $\frac{-y(1-x)}{1-y}$, so erhält man

$$
\begin{gathered}
A\left(-\frac{x(1-y)^{2}}{y(1-x)^{2}}\right)= \\
A\left(\frac{x(1-y)}{1-x}\right)+A\left(\frac{1-y}{y(1-x)}\right)+A\left(-\frac{x(1-y)}{y(1-x)}\right)+A\left(-\frac{1-y}{1-x}\right)-\frac{1}{2}(l y)^{2}+C .
\end{gathered}
$$

Addirt man jetzt diese beiden Formeln und die ursprüngliche (2) und führt die Vereinfachungen aus, welche die Formel (3) gewährt, so erhält man

$$
\begin{aligned}
& \text { 13. } A(-x y)+A\left(-\frac{x}{y}\right)+A\left(-\frac{x(1-y)^{2}}{y(1-x)^{2}}\right)= \\
& 2 A(-x)+2 A\left(-\frac{x(1-y)}{y(1-x)}\right)+2 A\left(\frac{x(1-y)}{1-x}\right)+C,
\end{aligned}
$$

und es ist $C=0$, mit Ausnahme des Falles, wenn $y$ und $1-x$ beide negativ sind, für welchen Fall $C=-2 \pi^{2}$ ist. Setzt man in dieser Formel $x=\operatorname{tang} \alpha \cdot \operatorname{tang} \beta, y=-\frac{\operatorname{tang} \alpha}{\operatorname{tang} \beta}$, so nimmt sie folgende Gestalt an :

14. $A\left(\operatorname{tang}^{2} \alpha\right)+\Lambda\left(\operatorname{tang}^{2} \beta\right)+A\left(\operatorname{tang}^{2}(\alpha+\beta)\right)=$ $2 A($ - tang $\alpha \operatorname{tang} \beta)+2 A($ - tang $\alpha \operatorname{tang}(\alpha+\beta))+2 A(-\operatorname{tang} \beta \operatorname{tang}(\alpha+\beta))+C$, wo $C=0$ ist, mit Ausnahme des Falles, dafs tang $\alpha$ und $\operatorname{tang} \beta$ gleiche Vorzeichen haben und tang $(\alpha+\beta)$ das entgegengesetzte von dem wenn $C=-2 \pi^{2}$ ist. Einen einfachen speciellen Fall der Formel (13) erhält man, wenn man $y=-1$ setzt, nämlich 
15. $A\left(\frac{4 x}{(1-x)^{2}}\right)=4 . A\left(\frac{2 x}{1-x}\right)+2 A(-x)-2 A(x)+C ;$ wo $C=0$ wenn $1-x$ positiv und $C=-2 \pi^{2}$ wenn $1-x$ negativ ist.

Alle bisher gefundenen Formeln haben wir dadurch abgeleitet, dafs wir in dem Integrale, welches die Function $\Lambda(x)$ darstellt, statt $x$ eine rationale gebrochene Function ersten Grades substituirt und das Integral sodann nach der allgemeinen Methode der Zerlegung in mehrere andere zerfället haben. Wir haben ferner bemerkt, dafs die Methode mit demselben Erfolge auch angewendet werden kann, wenn für $x$ irgend eine rationale Function von höherem Grade gesetzt wird. Wählt man hierzu die allgemeine Form einer rationalen gebrochenen Function zweiten Grades, so erhält man eine andere noch allgemeinere Formel als die Formel (2), in welcher, wenn die überflüssige, nur scheinbare Allgemeinheit, welche die vielen von einander unabhängigen Gröfsenzeichen anzeigen, durch passende Substitutionen aufgehoben wird, noch vier von einander unabhängige Quantitäten übrig bleiben, die aber nicht weniger als 25 besondere Functionen $A$ enthält. Beschränkt man sich hierbei auf speciellere Fälle, so vermindert sich zwar bei passender Bestimmung einer oder mehrerer der unabhängigen Grölsen die Anzahl der in der Formel enthaltenen Functionen $A$ beträchtlich: aber die einfachsten speciellen Fälle sind immer nur diejenigen, für welche die rationale Function zweiten Grades in eine ersten Grades übergeht; welche Fälle also schon in den hier gefundenen Formeln enthalten sein müssen. Wir wollen deshalb diese Formel, welche eine rationale Function zweiten Grades giebt, und um so mehr die höheren Graden entsprechenden übergehen und es bei den oben gefundenen Formeln, welche die einfachsten Eigenschaften der Function $\Lambda(x)$ ausdrücken, bewenden lassen.

\section{3.}

Wir haben nun noch zu zeigen, auf welche Weise die Werthe der Function $A(x)$ zu berechnen sind, und wollen deshalb zunächst den Gang dieser Function genauer untersuchen. Da der erste Differenzialquotient derselben, $\frac{l( \pm x)}{1+x}$ von $x=-\infty$ bis $x=+1$ negativ ist, von $x=+1$ bis $x=+\infty$ aber positiv; so nimmt die Function $A(x)$ in dem ganzen ersten Intervalle continuirlich ab; in dem zweiten Intervalle aber nimmt sie con- 
tinuirlich zu, und für $x=1$ hat sie ihr Minimum $A(1)=-\frac{\pi^{2}}{12}$ erreicht. Da ferner $A(0)=0$ ist, so folgt, dafs $A(x)$ für alle negativen Werthe des $x$ positiv, für positive Werthe des $x$ aber $\Lambda(x)$ anfänglich negativ ist, bis es, wenn $x$ wächst, wieder positiv wird und dann mit $x$ zugleich ins unendliche wächst. Für sehr grofse positive Werthe des $x$ ist nämlich $A(x)$ sehr nahe gleich $\frac{1}{2}(l x)^{2}-\frac{\pi^{2}}{6}$, und für sehr grolse negative Werthe des $x$ sehr nahe gleich $\frac{1}{2}(l-x)^{2}+\frac{\pi^{2}}{3}$; wie unmittelbar aus der Formel (3) hervorgeht. Damit man sich von dem Gange dieser Function eine genauere Vorstellung bilden könne, haben wir folgende Werthe derselben berechnet.

$$
\begin{aligned}
& \Lambda(0)=0,00000000000 \text {. } \\
& \Lambda(-1)=1,64493406685 \text {. } \\
& \Lambda(1)=-0,82246703342 \text {. } \\
& \Lambda(2)=-0,67524635647 \text {. } \\
& \Lambda(3)=-0,41637539993 \text {. } \\
& \Lambda(4)=-0,13878509442 \text {. } \\
& A(5)=+0,13444649368 \text {. } \\
& A(6)=+0,39666088475 \text {. } \\
& A(-2)=2,46740110027 \text {. } \\
& A(-3)=3,08168043373 \text {. } \\
& A(-4)=3,58430948761 \text {. } \\
& A(-5)=4,01487386385 \text {. } \\
& A(-6)=4,39421319291 \text {. } \\
& A(7)=+0,64624435589 \text {. } \\
& A(-7)=4,73487611794 \text {. } \\
& \Lambda(8)=+0,883324061 \% 6 \text {. } \\
& A(-8)=5,04509611128 \text {. } \\
& A(9)=+1,10863277949 \text {. } \\
& \Lambda(-9)=5,33061006760 \text {. } \\
& \Lambda(10)=+1,32308002285 \text {. } \\
& A(-10)=5,59559784509 \text {. } \\
& \boldsymbol{A}(-11)=5,84321195371 \text {. }
\end{aligned}
$$

Der zweite Werth des $x$, für welchen $A(x)=0$ wird, liegt, wie man aus dieser Tabelle sieht, zwischen 4 und 5: derselbe ist, wie wir durch genaue Rechnung gefunden haben, $A(x)=0$ für $x=4,50374185563$.

Die Berechnung der numerischen Werthe der Function $\mathcal{A}(x)$ wird durch die oben gefundenen Formeln aufserordentlich erleichtert. Zunächst zeigen dieselben, wie alle diese Functionen sich auf andere reduciren lassen, für welche $x$ in dem Intervalle $x=0$ bis $x=-\frac{1}{2}$, oder auch in dem Intervalle $x=-\frac{1}{2}$ bis $x=-1$ liegt. Durch die Formel (3) kann man zunächst jede Function $A(x)$, in welcher $x$, vom Vorzeichen abgesehen, grörser als 1 ist, in eine andere verwandeln, in welcher $x$ kleiner als 1 ist; es bleibt also nur das Intervall $x=-1$ bis $x=+1$. Durch die Formel (4) wird ferner jede Function $A(x)$, in welcher $x$ in den Grenzen $x=+1$ bis $x=0$ liegt, in eine andere verwandelt, in welcher $x$ in den Grenzen $x=-1$ bis $x=0$ liegt; und dieses Intervall wird endlich durch 
die Formel (5) auf die Hälfte reducirt, so dafs man also die Function $A(x)$ nur für diejenigen Werthe des $x$ besonders zu berechnen hat, welche in den Grenzen $x=-\frac{1}{2}$ bis $x=0$ liegen. Das Intervall - $\frac{1}{2}$ bis 0 lärst sich vermittelst der gefundenen Formeln weiter einschränken, auf $-\frac{1}{3}$ bis 0 , dieses wieder auf $-\frac{1}{4}$ bis 0 und dieses wieder auf $-\frac{1}{5}$ bis 0 und so fort in's unendliche: oder es läfst sich jede Function $A(x)$ durch andere solche Functionen ausdrücken, deren Elemente negativ sind und von 0 so wenig verschieden, als man nur will. Um dies zu zeigen, nehme ich die Formel $A\left(-x^{2}\right)=2 A(-x)+2 A(x)$ und verwandele in derselben $A(-x)$ nach der Formel (4), wodurch ich erhalte

$$
\text { 16. } \quad \Lambda(x)=\Lambda\left(\frac{x}{1-x}\right)+\frac{1}{2} A\left(-x^{2}\right)-\frac{1}{2}(\Lambda(1-x))^{2} \text {, }
$$

wenn $1-x$ positiv ist. Mit Hülfe dieser Formel wird jede Function $A(x)$, deren Element $x$ in den Grenzen $-\frac{1}{2}$ und $-\frac{1}{3}$ liegt, durch zwei andere ausgedrückt, deren Elemente in den Grenzen - $-\frac{1}{3}$ und 0 liegen. Ferner wird durch dieselbe Formel jede Function $A(x)$, deren Element $x$ in den Grenzen $-\frac{1}{3}$ und $-\frac{1}{4}$ liegt, durch zwei andere ausgedrückt, deren Elemente in den Grenzen $-\frac{1}{4}$ und 0 liegen. Von diesem Intervalle wird wieder der Theil zwischen $-\frac{1}{4}$ und $-\frac{1}{5}$ durch den anderen Theil bestimmt, und es werden so nach einander noch von dem Intervalle $-\frac{1}{5}$ bis 0 die einzelnen Intervalle $-\frac{1}{5}$ bis $-\frac{1}{6},-\frac{1}{6}$ bis $-\frac{1}{9}$ u. s. w. abgesondert, so dafs nur das Intervall $-\frac{1}{n}$ bis 0 bleibt, in welchem man $n$ so grofs machen kann, als man will. Es liefse sich, wie man leicht sieht, hierauf eine Methode der näherungsweisen Berechnung der Function $A(x)$ gründen, welche jedoch von keinem practischen Nutzen sein würde, da die Annäherung an den Grenzwerth $\Lambda(0)$ viel zu langsam ist. Eine oder zwei solche Reductionen aber, welche das Element $x$ der zu berechnenden Function kleiner als $-\frac{1}{3}$ oder kleiner als $-\frac{1}{4}$ machen, werden in vielen Fällen förderlich sein, da die Function $A(x)$ am leichtesten durch eine einfache, nach Potenzen von $x$ geordnete Reihe berechnet wird.

Um die Reihen-Entwickelungen des Integrals $A(x)$ zu erhalten, verwandle ich dasselbe durch theilweise Integration in

$$
A(x)=l x l(1+x)-\int \frac{d x}{x} l(1+x) .
$$

Wird nun $l(1+x)$ in eine Reihe entwickelt und die Integration ausgeführt, so erhält man

Crelle's Journal d. M. Bd. XXI. Hft. 1. 


$$
\text { 17. } \quad A(x)=l x l(1+x)-\left(\frac{x}{1^{2}}-\frac{x^{2}}{2^{2}}+\frac{x^{3}}{3^{2}}-\ldots .\right)
$$

in den Grenzen $x=-1$ bis $x=+1$. Aus dieser Reihen-Entwickelung erhält man sogleich noch fünf andere, indem man mit Hülfe der fünf einfachen Gleichungen ( 3 bis 7, \$. 2.) die Function $\Lambda(x)$ umformt und alsdann durch eine passende Substitution für $x, A(x)$ wieder herstellt. Diese Reihen sind

18. $\quad A(x)=K+l x l(1+x)-\frac{1}{2}(l x)^{2}+\left(\frac{1}{1^{2} x}-\frac{1}{2^{2} x^{2}}+\frac{1}{3^{3} x^{3}}-\ldots\right)$, wo $K=-\frac{\pi^{2}}{6}$ ist, in den Grenzen $x=+1$ bis $x=+\infty$, und $K=+\frac{\pi^{2}}{3}$ in den Grenzen $x=-\infty$ bis $x=-1$;

19. $\quad A(x)=\frac{\pi^{2}}{6}-\left(\frac{1+x}{1^{2}}+\frac{(1+x)^{2}}{2^{2}}+\frac{(1+x)^{3}}{3^{2}}+\ldots.\right)$ in den Grenzen $x=-2$ bis $x=0$;

20. $A(x)=K+\frac{1}{2}(l(1+x))^{2}+\left(\frac{1}{1+x}+\frac{1}{2^{2}(1+x)^{2}}+\frac{1}{3^{2}(1+x)^{3}}+\cdots\right)$, wo $K=-\frac{\pi^{2}}{6}$ ist, in den Grenzen $x=0 \quad$ bis $x=\infty$, und $K=+\frac{\pi^{2}}{3}$ in den Grenzen $x=-\infty$ bis $x=-2$; 21. $A(x)=$ $l x l(1+x)-\frac{1}{2}(l(1+x))^{2}-\left(\frac{x}{1+x}+\frac{x^{2}}{2^{2}}(1+x)^{2}+\frac{x^{3}}{3^{2}(1+x)^{3}}+\ldots\right)$ in den Grenzen $x=-\frac{1}{2}$ bis $x=+\infty$;

22. $\quad A(x)=\frac{\pi^{2}}{6}+\frac{1}{2}(l-x)^{2}+\left(\frac{1+x}{x}+\frac{(1+x)^{2}}{2^{2} x^{2}}+\frac{(1+x)^{3}}{2^{3} x^{3}}+\ldots.\right)$ in den Grenzen $x=-\infty$ bis $x=-\frac{1}{2}$.

Diese Reihen-Entwickelungen dienen zur unmittelbaren Berechnung jder Function $A(x)$ für alle möglichen Werthe des $x$, und zwar dienen die Reihen (17) und (21) vorzüglich für den Fall, wenn $x$ den Werthe 0 nahe liegt; die Reihen (19) und (22) für den Fall, wenn $x$ dem Werthe -1 nahe liegt, und die Reihen (18) und (20) für grofse posilive und negative Werthe des $x$.

(Die Fortsetzung folgt im nächsten Hefte.); 